\title{
Downward Control from the Lower Stratosphere?
}

\author{
JOSEPH EGGER \\ Meteorologisches Institut, Universität München, Munich, Germany \\ Klaus-Peter Hoinka \\ Institut für Physik der Atmosphäre, DLR, Oberpfaffenhofen, Germany
}

\begin{abstract}
The concept of downward control proposes a mechanism for the impact of the stratospheric circulation on the troposphere. Momentum forcing at upper-stratospheric levels induces a meridional circulation that eventually reaches the surface. So far, a lack of sufficiently accurate data hindered an observational test of this downward propagation. The concept is extended in this paper by looking at the effect of angular momentum forcing in prescribed regions in the lower stratosphere on the tropospheric circulation. In that case, the European Centre for Medium-Range Weather Forecasts Reanalysis Project (ERA) data can be used to investigate the atmospheric response to forcing in a prescribed domain. It is found that these forcing events are quite short lived and that angular momentum flux convergence in the prescribed domain is highly correlated with convergence outside this forcing area. Typically, these fields of convergence and also divergence extend to the surface in a quasibarotropic manner outside the Tropics. This structure of the forcing is not compatible with the assumptions of the downward control concept. The observed related meridional circulation therefore differs widely from that predicted. In particular, there is no obvious descent of the circulation to the ground. Even so, such forcing events are accompanied by an intensive exchange of angular momentum between stratosphere and troposphere. The confinement of the forcing to the selected forcing domain is reasonably strict in the Tropics. A relatively narrow tongue of angular momentum is growing at the equator underneath the forcing area. Frictional torques play a role in this development. Altogether, the forcing events as selected involve a strong angular momentum exchange between stratosphere and troposphere but are not suited for a test of the downward control concept. Alternatives are discussed.
\end{abstract}

\section{Introduction}

The mechanism of downward control (Haynes et al. 1991, hereafter H91) is concerned with the impact of the stratosphere on the troposphere via momentum forcing $G$ at upper-stratospheric levels. This anomalous wave driving acts, for example, in the approximate zonal momentum equation

$$
\frac{\partial \bar{u}}{\partial t}-2 \Omega \sin \varphi \bar{v}=\bar{G},
$$

where

$$
\bar{u}=\int_{0}^{2 \pi} u a \cos \varphi d \lambda
$$

Corresponding author address: Joseph Egger, Meteorologisches Institut, Universität München, Theresienstrasse 37, $80333 \mathrm{Mu}-$ nich, Germany.

E-mail: J.Egger@lrz.uni-muenchen.de is the zonal integral of the zonal wind velocity and $\bar{v}$ is the corresponding meridional component (H91; $\Omega=$ $2 \pi$ day $^{-1} ; \varphi$ latitude; $\lambda$ longitude). Given the switch on of a wave-induced force $\bar{G}$ restricted to a forcing area $\mathrm{F}$ in the upper stratosphere, H91 showed analytically and numerically that a meridional circulation and corresponding changes of the zonal wind are induced mainly below $\mathrm{F}$ but there is also a weak response propagating upward (see also Holton et al. 1995). Eddy feedbacks in the troposphere may alter and enhance the response (e.g., Song and Robinson 2004). Although the underlying processes appear to be robust and well understood it is not clear as yet if this mechanism is capable of inducing substantial accelerations in the troposphere. The stratospheric momentum changes at heights of, say, 40-50 km (H91; Holton et al. 1995) may be too small to generate appreciable anomalies of the zonal wind at low levels [Egger (1996); see Shepherd and Shaw (2004) for further constraints]. On the other hand, numerical experiments with anomalous wave 
driving at 25-35 km (Song and Robinson 2004) resulted in clear responses in the troposphere. Lack of sufficiently accurate data at upper levels so far has prevented tests of the concept with respect to flow evolution in time. Matters are different if we look at events of momentum forcing in the lower stratosphere where such data are available. Forcing areas $\mathrm{F}$ can be specified in the lower stratosphere, and the tropospheric and stratospheric response to observed forcing $G$ in F can be analyzed. In particular, we look for the evolution of stratospheric impacts on the troposphere.

Following H91, we reformulate this problem in terms of the axial angular momentum equation

$$
\begin{aligned}
\frac{\partial}{\partial t} m \rho & +(a \cos \varphi)^{-1} \frac{\partial}{\partial \lambda}(u \rho m)+(a \cos \varphi)^{-1} \frac{\partial}{\partial \varphi}(v \rho m \cos \varphi) \\
& +\frac{\partial}{\partial z}(\rho w m)=-\frac{\partial p}{\partial \lambda},
\end{aligned}
$$

where

$$
m=(u+\Omega a \cos \varphi) a \cos \varphi
$$

is the specific axial angular momentum and $p$ is pressure. Stress terms are deleted in (1.3) as being unimportant well above the ground. Zonal integration of (1.3) gives

$$
\frac{\partial}{\partial t} \bar{\mu}+a^{-1} \frac{\partial}{\partial \varphi} V+\frac{\partial}{\partial z} W=0
$$

above the mountains. In (1.5), $\bar{\mu}=\overline{m \rho}$ is the zonally integrated angular momentum and

$$
\begin{aligned}
V & =\overline{v m \rho}=V_{m}+V_{w} \quad \text { and } \\
W & =\overline{w m \rho}=W_{m}+W_{w}
\end{aligned}
$$

are the mean fluxes. These are split in a flux $V_{m}$ of the mass term

$$
\mu_{m}=\rho \Omega a^{2} \cos ^{2} \varphi
$$

and a flux $V_{w}$ of the wind term

$$
\mu_{w}=\rho и a \cos \varphi
$$

where, of course, $\mu=\mu_{m}+\mu_{w}$. Corresponding definitions apply in (1.7). Hence, (1.5) can be replaced by

$$
\frac{\partial}{\partial t} \bar{\mu}+a^{-1} \frac{\partial}{\partial \varphi} V_{m}+\frac{\partial}{\partial z} W_{m}=-\bar{a}^{-1} \frac{\partial}{\partial \varphi} V_{w}-\frac{\partial}{\partial z} W_{w} .
$$

Let us introduce the divergences

$$
D_{m}=a^{-1} \frac{\partial}{\partial \varphi} V_{m}+\frac{\partial}{\partial z} W_{m} \text { and }
$$

$$
D_{w}=a^{-1} \frac{\partial}{\partial \varphi} V_{w}+\frac{\partial}{\partial z} W_{w}
$$

of the mass and wind-term fluxes. The first term on the left-hand side in (1.10) is analogous to the tendency of the zonal wind in (1.1). The sum $D_{m}$ of the second and third term is approximated by the Coriolis term in (1.1) (see also 1.16). The term $D_{w}$ on the right-hand side of (1.10) generalizes the forcing $\bar{G}$ in (1.1). The final step is to select a forcing area $\mathrm{F}$ for which

$$
G_{F}=-\int_{\mathrm{F}} D_{w} d f
$$

is the integral of the convergence of the wind-term fluxes over F. For example, the forcing areas selected by H91 and Holton et al. (1995) may be chosen in (1.13) so that $G_{F} \sim \bar{G}$.

It is common to analyze the atmospheric response to a given forcing within the frame work of the transformed Eulerian-mean equations (e.g., Andrews et al. 1987) where (1.1) is replaced by

$$
\frac{\partial \bar{u}}{\partial t}-2 \Omega \sin \varphi \bar{v}^{x}=-\nabla \cdot \mathbf{F}
$$

with residual meridional velocity $v^{x}$ and the EliassenPalm flux $\mathbf{F}$ in the simplest case. In particular, $\bar{G}$ could be absorbed into the divergence of the Eliassen-Palm flux. It appears, however, preferable to use (1.10) because (1.14) obscures angular momentum conservation, a feature of key importance to our approach.

It is the purpose of this paper to evaluate the atmospheric response to a forcing (1.13) in selected regions on the basis of data. Note, in particular, that we are concerned here with the time-dependent part of the work of H91. Steady-state aspects will not be dealt with. Clearly, this is a statistical problem where we have to look at many cases to obtain a reliable answer. That can be done by selecting events with forcing in a given area, calculating the terms in (1.10) for every event and evaluating finally the covariances of forcing and response. It is, however, easier and more elegant to determine first these covariances of forcing and response on the basis of the observations and insert those in a statistical version of (1.10) where just these covariances are the variables. Both methods give the same result, of course.

It is straightforward to derive this statistical version of (1.10) (e.g., Papoulis 1965). Let $C(s, p \mid \tau)$ the covariance function of a variable $s$ with another variable $p$ as a function of lag $\tau$. Multiplication of (1.10) by $G_{F}(\tilde{t})$ with $\tilde{t}=t-\tau$ gives

$$
\begin{aligned}
G_{F}(\tilde{t}) \frac{\partial}{\partial \tau} \bar{\mu}(\tilde{t}+\tau) & +G_{F}(\tilde{t}) D_{m}(\tilde{t}+\tau) \\
& =-G_{F}(\tilde{t}) D_{w}(\tilde{t}+\tau) .
\end{aligned}
$$


After taking expectations results

$$
\frac{\partial}{\partial \tau} C\left(G_{F}, \bar{\mu} \mid \tau\right)+C\left(G_{F}, D_{m} \mid \tau\right)=-C\left(G_{F}, D_{w} \mid \tau\right)
$$

as the equation to be analyzed in the remainder on the basis of data. The first term on the left-hand side describes the evolution of the covariance of the forcing $G_{F}$ and the atmospheric angular momentum with lag. It is a generalization of the tendency in (1.1). The second one is essentially the covariance function of forcing and the Coriolis term. To see this more clearly we may rewrite (1.1) in the form

$$
\frac{\partial \bar{u} \cos \varphi}{\partial t}+\frac{\partial}{\partial \varphi} \Omega \cos ^{2} \varphi \bar{v}+\rho_{o}^{-1} \frac{\partial}{\partial z} \rho_{o} \Omega a \cos ^{2} \varphi \bar{w}=\bar{G} \cos \varphi
$$

so that indeed the Coriolis term is seen to stand for the divergence of the mass term flux. The term $A=$ $-C\left(G_{F}, D_{w} \mid \tau\right)$ on the right-hand side of (1.15) corresponds, of course, with the term $\bar{G} \cos \varphi$ in (1.16), but while $\bar{G}$ vanishes outside $\mathrm{F}$, this is not generally true with respect to $A$. Of course, the term $A$ is large in $\mathrm{F}$ at least for small lags because of (1.13). There is, however, no reason why $A$ should vanish outside F. Divergences $D_{w}$ in other parts of the atmosphere may be statistically related to $G_{F}$ so that $A \neq 0$ there. The forcing $\bar{G}$ is specified in separation in the cases assumed by H91. It is not obvious if the data contain such cases.

Altogether, at least three different types of results are conceivable for this test. We may find a sufficient number of events where the assumptions of H91 are sufficiently satisfied. In that case we expect to see vertical angular momentum transports evolve in response to the forcing as predicted by the theory of downward control (H91). If the results support this expectation, the theory is corroborated, if not, one has to search for reasons for this failure. Third, we may not be able to find cases where the theory can be applied.

It is customary to characterize the interaction of troposphere and stratosphere by the exchanges of air mass, water vapor, and trace substances (e.g., Hoerling et al. 1993; Wirth and Egger 1999; Wernli and Bourqui 2002). The calculations of H91 suggest that forcing events are also be accompanied by such exchanges. We expect, in particular, that angular momentum will be transferred. Such transfers will be quantified as a byproduct of the tests.

\section{Data and strategy}

The data to be used are part of the dataset covering the period January 1979-December 1992 generated by the European Centre for Medium-Range Weather Forecasts Reanalysis Project (ERA; Gibson et al. 1997). Wind and density are available at the 31 levels of the hybrid $\sigma$ system of the ERA scheme. Torques can be calculated as well on the basis of the ERA data. All data are transformed to equidistant $z$ surfaces with a vertical spacing $\Delta z=1000 \mathrm{~m}$. The representation of the mass terms as function of height is possible only in a spatially fixed system. Latitude belts of width $a \Delta \varphi=$ $1000 \mathrm{~km}$ are introduced as well. The fluxes $V$ and $W$ are evaluated such that angular momentum budgets can be evaluated for all annuli (i,j) of width

$$
-\frac{\pi}{2}+(i-1) \Delta \varphi \leq \varphi \leq-\frac{\pi}{2}+i \Delta \varphi
$$

and depth

$$
(j-1) \Delta z \leq z \leq j \Delta z .
$$

In particular, (1.12) is integrated over the volume of an annulus so that

$$
\begin{aligned}
& \frac{d C\left(G_{F}, \tilde{\mu}_{i j} \mid \tau\right)}{d \tau}+C\left[G_{F},\left(\tilde{V}_{i+1, j}-\tilde{V}_{i, j}+\tilde{W}_{i, j+1}-\tilde{W}_{i, j}\right) \mid \tau\right] \\
& \quad=0
\end{aligned}
$$

where $\tilde{\mu}_{i j}$ is the integral of $\bar{\mu}$ over the area of the annulus and $\tilde{V}_{i+1 j}\left(\tilde{W}_{i j+1}\right)$ is the integral of $V=V_{m}+V_{w}$ ( $W=W_{m}+W_{v}$ ) over the northern (upper) boundary of the annulus. Of course, these transports may be split in mass and wind-term contributions. Forcing areas are characterized by the index intervals $I_{s} \leq i \leq I_{n}, J_{u} \leq j$ $\leq J_{o}$, so that

$$
G_{F}=-\sum_{j=J_{u}}^{J_{o}}\left(\tilde{V}_{I_{n+1}, j}-\tilde{V}_{I_{s}, j}\right)-\sum_{i=I_{s}}^{I_{n}}\left(\tilde{W}_{i, J_{o+1}}-\tilde{W}_{i, J_{u}}\right) .
$$

Although the $z$ surfaces of our grid are evenly spaced up to a height of $28 \mathrm{~km}$, those of the ERA scheme are not. There are only few analysis surfaces left above a height of $20 \mathrm{~km}$. It has been decided, therefore, to only consider forcing areas $G_{F}$ below heights of $21 \mathrm{~km}$, that is, in the lower stratosphere. The annual and semiannual cycle is suppressed by subtracting the corresponding Fourier modes. The available four daily values per variable in the ERA set are averaged over the day. Further details on the data handling are found in Egger and Hoinka (2005).

It is useful (see Egger and Hoinka 2005) to introduce a generalized streamfunction $\psi$ and velocity potential $\chi$ per annulus $(i, j)$ via

$$
\begin{gathered}
C\left(G_{F}, \tilde{V}_{i, j} \mid \tau\right)=\psi_{i, j}-\psi_{i, j+1}+\chi_{i+1, j}-\chi_{i, j}, \\
C\left(G_{F}, \tilde{W}_{i, j} \mid \tau\right)=\psi_{i+1, j}-\psi_{i, j}+\chi_{i, j+1}-\chi_{i, j} .
\end{gathered}
$$


Given the observed covariance functions at the left, it is straightforward to determine $\psi$ and $\chi$ where the streamfunction represents that part of the angular momentum fluxes that has no impact on changes of the angular momentum and which are captured by the velocity potential. At the ground $(j=1)$ we have

$$
C\left(G_{F}, \tilde{W}_{i 1} \mid \tau\right)=C\left(G_{F}, T_{i} \mid \tau\right),
$$

where $T_{i}=T_{f i}+T_{m i}$ is the sum of the friction and mountain torque per belt with

$$
\begin{aligned}
T_{f i} & =\int_{s_{i}} \tau_{x} a \cos \varphi d s_{i}, \\
T_{m i} & =\int_{s_{i}} h \frac{\partial p_{s}}{\partial \lambda} d s_{i}
\end{aligned}
$$

( $\tau_{x}$ zonal surface stress, $h$ topography, $p_{s}$ surface pressure, $s_{i}$ surface of belt $i$ ). These torques are not included in (1.6) because this equation is strictly valid only above the top of the earth's mountains. A more complete version of this equation is given in Egger and Hoinka (2005) where the torques represent the transfer of angular momentum at the earth's surface. If all terms $C\left(G_{F}, T_{i} \mid \tau\right)$ are of the same sign, clearly,

$$
\begin{aligned}
\chi_{i 2}-\chi_{i 1} & =C\left(G_{F}, T_{i} \mid \tau\right) \\
\psi_{i 1} & =0
\end{aligned}
$$

is the boundary condition to be posed. If the sign varies we assume that only those parts of the torques contribute to $\chi_{\mathrm{ij}}$, which cannot be balanced by torques of opposite sign in another belt. Details of the procedure are discussed in Egger and Hoinka (2005). Note that a splitting of $\psi$ and $\chi$ in mass and wind-term flux contributions is not meaningful because there are no boundary conditions available in this case.

\section{Results}

A reasonably good coverage of our problem is achieved by selecting forcing areas in midlatitudes, the polar caps, and in the Tropics.

\section{a. Lower stratosphere at midlatitudes}

The forcing area in case $\mathrm{M}_{1}$ covers the domain $27^{\circ} \mathrm{N}$ $\leq \varphi \leq 63^{\circ} \mathrm{N}, 11 \mathrm{~km} \leq z \leq 14 \mathrm{~km}$ in the lower stratosphere $\left(I_{s}=14, I_{n}=17 ; J_{u}=12, J_{o}=15\right.$; see also Table $1)$. This size of the forcing area is similar to that specified in Holton et al. (1995). The autocovariance function $C\left(G_{F}, G_{F} \mid \tau\right)$ for this domain is displayed in Fig. 1. There is a sharp peak of $46 \mathrm{Ha}^{2}$ amplitude at $\tau=0$. The first zero crossing occurs at a lag $\sim 5$ days but there is a
TABLE 1. List of cases discussed in the text. The widths (depths) of the forcing domains are given in the second (third) column.

\begin{tabular}{lcc}
\hline \hline Case & \multicolumn{2}{c}{ Forcing domain } \\
\hline $\mathrm{M}_{1}$ & $27^{\circ} \mathrm{N} \leq \varphi \leq 63^{\circ} \mathrm{N}$ & $11 \leq \mathrm{z} \leq 14 \mathrm{~km}$ \\
$\mathrm{M}_{2}$ & $27^{\circ} \mathrm{N} \leq \varphi \leq 63^{\circ} \mathrm{N}$ & $14 \leq \mathrm{z} \leq 17 \mathrm{~km}$ \\
$\mathrm{M}_{3}$ & $27^{\circ} \mathrm{N} \leq \varphi \leq 36^{\circ} \mathrm{N}$ & $17 \leq \mathrm{z} \leq 20 \mathrm{~km}$ \\
$\mathrm{M}_{4}$ & $63^{\circ} \mathrm{S} \leq \varphi \leq 27^{\circ} \mathrm{S}$ & $11 \leq \mathrm{z} \leq 17 \mathrm{~km}$ \\
$\mathrm{P}_{1}$ & $63^{\circ} \mathrm{N} \leq \varphi \leq 90^{\circ} \mathrm{N}$ & $11 \leq \mathrm{z} \leq 14 \mathrm{~km}$ \\
$\mathrm{~T}_{1}$ & $-18^{\circ} \mathrm{S} \leq \varphi \leq 18^{\circ} \mathrm{N}$ & $13 \leq \mathrm{z} \leq 16 \mathrm{~km}$ \\
\hline
\end{tabular}

small secondary maximum for lag $\tau=17$ days. Thus, strong events of convergence are short lived, but there appears to exist also a class of weak long-term events.

The response is not restricted to the forcing area even at $\tau=0$ (Fig. 2a) but extends to the surface with a maximum near the ground and another one in the forcing area $\mathrm{F}$. The reaction is slightly stronger for positive lags of a few days. The covariance field $C\left(G_{F}, \tilde{\mu} \mid \tau\right)$ for $\tau=4$ days (Fig. 2b) differs relatively little from that at $\tau=0$. To facilitate the discussion we assume in the following that $G_{F}$ is positive at $\tau=0$. The patterns in Fig. 2 is quasibarotropic with positive values of $\tilde{\mu}$ in the forcing area and underneath and negative values in the south. Obviously, angular momentum is transported out of the southern belts and deposited in the north during the forcing events. These transports extend well into the stratosphere. This pattern is fairly long lived when compared to the decay of $G_{F}$ in Fig. 1. The basic features of this pattern are seen even for $\tau=-8$ days but amplitudes are just one-third of those in Fig. 2.

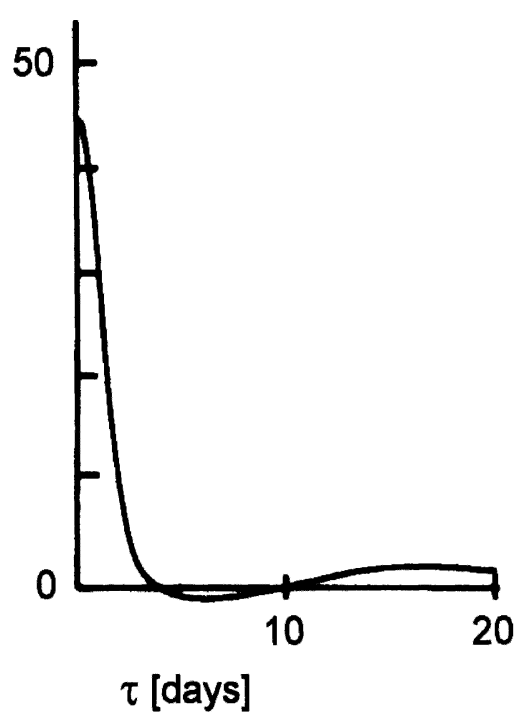

FIG. 1. Autocovariance function of $G_{F}$ in $\mathrm{Ha}^{2}(1 \mathrm{Hadley}=1 \mathrm{Ha}$ $\left.=10^{18} \mathrm{~kg} \mathrm{~m}^{2} \mathrm{~s}^{-2}\right)$ for the forcing area $27^{\circ} \mathrm{N} \leq \varphi \leq 63^{\circ} \mathrm{N}, 11 \mathrm{~km} \leq$ $z \leq 14 \mathrm{~km}\left(I_{s}=14, I_{n}=17 ; J_{u}=12, J_{\mathrm{o}}=18\right)$ of $\mathrm{M}_{1}$ as a function of lag $\tau$ in days. 

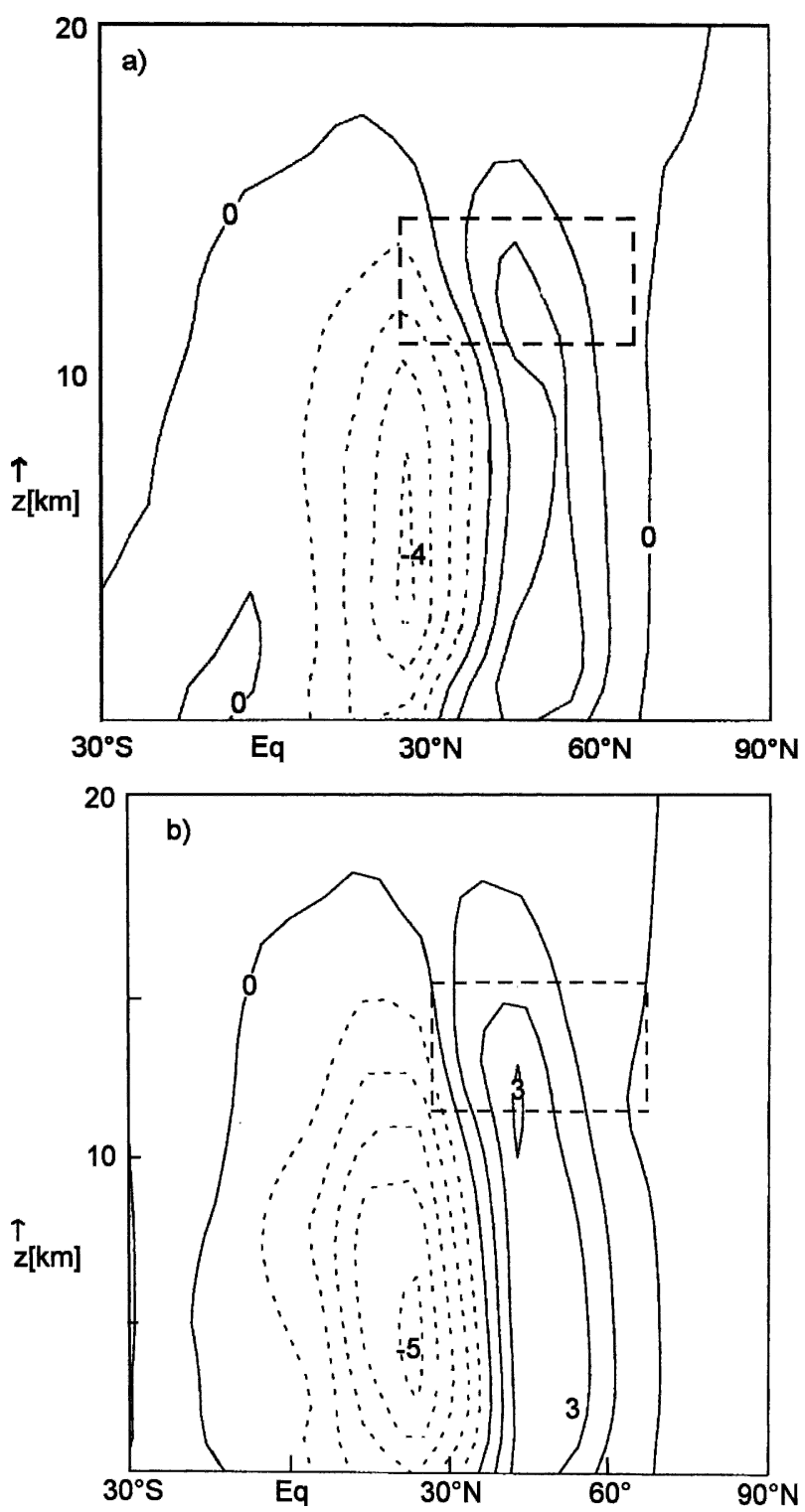

FIG. 2. Covariance function $C\left(G_{F}, \tilde{\mu} \mid \tau\right)$ in $\mathrm{Ha}^{2} \mathrm{~s}^{-1}$ at lag (a) $\tau=$ 0 days and (b) $\tau=4$ days in $\mathrm{M}_{1}$. Negative values dashed. The rectangle marks the forcing area. Contour interval is $10^{5} \mathrm{Ha}^{2} \mathrm{~s}^{-1}$.

Figure 2 suggests strongly that the forcing $G_{F}$ is part of a large-scale convergence of angular momentum due to the convergence of eddy momentum transports. Corresponding analyses by Feldstein and Lee (1998), Lorenz and Hartmann (2001), and many others demonstrated that these convergences have a quasibarotropic structure extending from the ground to the lower stratosphere and an autocovariance of similar shape to what is seen in Fig. 1. Indeed, the pattern of $C\left(G_{F}, D_{w} \mid \tau\right)$ at $\tau=0$ (not shown) is fairly similar to that in Fig. 2 except for the sign, of course. The covariance $C\left(G_{F}, D_{m} \mid \tau\right)$ is also negative in the forcing area and below (not shown) at $\tau=0$, so that the meridional mass transport is positive near the forcing area [see (1.15), (1.16), and the related discussion] in contrast to what would be expected from (1.1). This pattern decays, however, as quickly with lag as the forcing.

H91 predict the onset of negative torques for positive $G_{F}$ some time after the forcing has been switched on. That makes good sense because the forcing accelerates the atmosphere. Surface friction will eventually slow down the circulation, that is, a negative torque will be exerted. The covariance of $G_{F}$ with the global torque $T$ is indeed rapidly decreasing for $-1 \leq \tau \leq 6$ days (Fig. 3 ), but the resulting covariances are as large as $\sim 10 \mathrm{Ha}^{2}$ and, of course, cannot be totally explained by the action in the forcing area. The following increase to large positive values may reflect the impact of the tropical anomalies in Fig. 2.

The corresponding streamfunction and velocity potential patterns are displayed in Figs. 4 and 5. There is downward (upward) nondivergent flux of positive (negative) angular momentum at $\tau=0$ mainly below the forcing area with ascending branches to the north and south (Fig. 4). Three days before (not shown) the cyclonic cell is centered in the forcing area but with just one third of the amplitude in Fig. 4. Three days later the fluxes are weaker but similar to those in Fig. 4. The velocity potential at $\tau=0$ (Fig. 5a) is dominated by northward flow into the forcing domain and into the air column below. The flux near the tropopause $(z \sim 10-15$ $\mathrm{km}$ ) is positive. Given positive forcing the stratosphere receives angular momentum. Torques do not contribute at that time, as can be seen from the orientation of the isolines normal to the surface. Three days later (Fig. $5 \mathrm{~b}$ ), there is a maximum of $\chi$ below the forcing domain indicating a loss of stratospheric angular momentum in F. There is a negative torque event with flux into the ground. This event corresponds with the minimum in Fig. 3.

The evaluation of fluxes across the tropopause is fraught with uncertainties. The calculation of trajectories appears to be the most accurate method at the moment (e.g., Wernli and Bourqui 2002). This method is, however, not suited for angular momentum exchanges because angular momentum is not conserved during particle transport. Instead, we choose an extremely simple procedure to estimate vertical transports near the tropopause. Assume again that $G_{F}$ is positive. The total upward flux through the surface $z=$ $z_{j}$ is then given by

$$
F_{j}^{+}(\tau)=\frac{1}{2} \sum_{i}\left[C\left(G_{F}, \tilde{W}_{i, j} \mid \tau\right)+\left|C\left(G_{F}, \tilde{W}_{i, j} \mid \tau\right)\right|\right]
$$




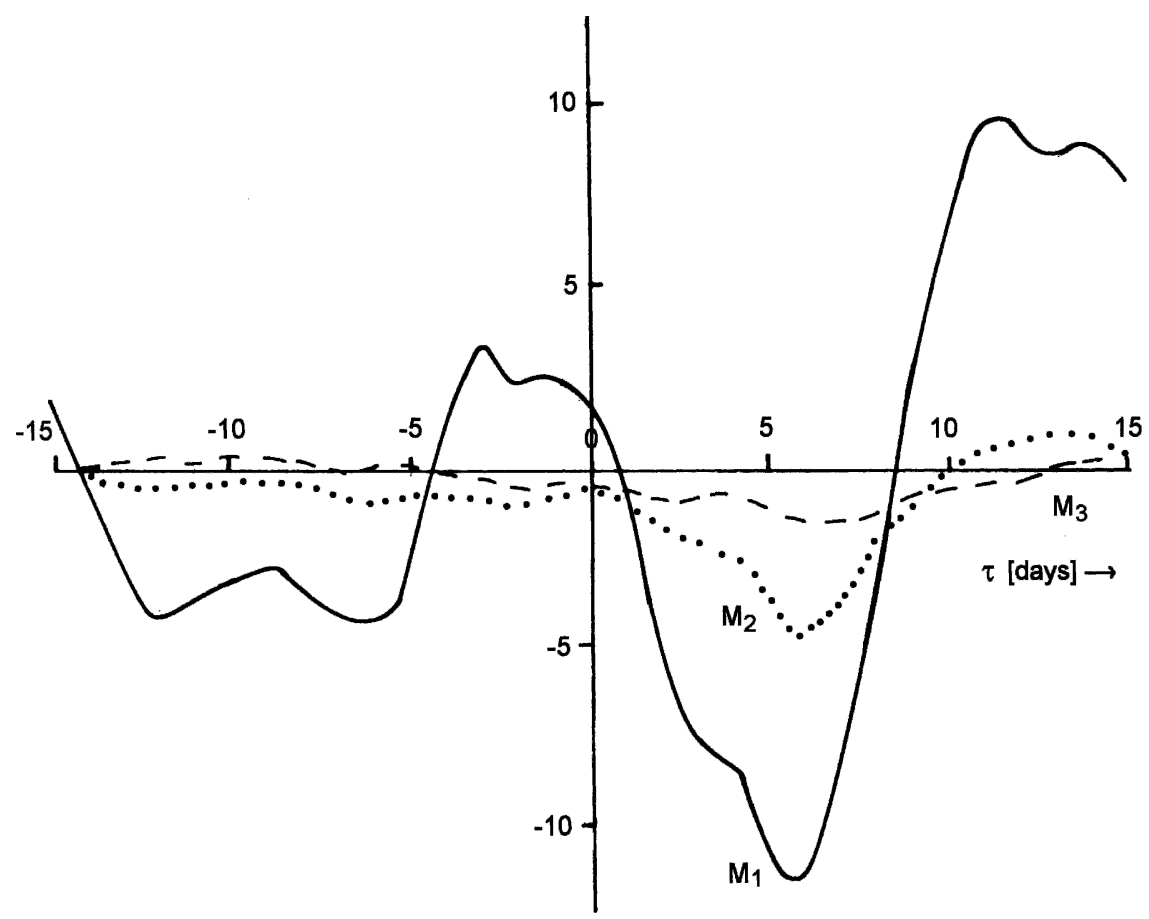

FIG. 3. Covariance function $C\left(G_{F}, T \mid \tau\right)$ of the forcing $G_{f}$ with the global torque in $\mathrm{Ha}^{2}$ for lags $-15 \leq \tau \leq 15$ days and for the three cases $\mathrm{M}_{1}$ (bold), $\mathrm{M}_{2}$ (dotted), and $\mathrm{M}_{3}$ (dashed).

The corresponding negative flux $F_{j}^{-}$is obtained by changing the sign in (3.1). These fluxes are listed in Table 2 for various levels and lags. There is a close balance of positive and negative contributions so that the sum of $F^{+}$and $F^{-}$is of the order $\leq 10 \mathrm{Ha}^{2}$. The fluxes are largest in the troposphere and decrease quickly with increasing height. To obtain a feeling for the accelerations involved we have to recall that a torque of $1 \mathrm{Ha}$ has to act for about 100 days to increase the factor $u_{o}$ in globally superrotating zonal flow $u_{o}$ $\cos \varphi$ by $1 \mathrm{~m} \mathrm{~s}^{-1}$. Figure 1 tells us that the standard deviation of $G_{F}$ is $\sim 7 \mathrm{Ha}$. Thus, if a value of $F^{+}$of 200 $\mathrm{Ha}^{2}$ is maintained for 2 days that implies an acceleration of a stratospheric superrotation by $\sim 6 \mathrm{~m} \mathrm{~s}^{-1}$ during that time assuming that the stratospheric mass is one tenth of the total mass of the atmosphere. However, most of this flux does not affect the angular momentum of the stratosphere but is nondivergent. It is, perhaps, more relevant to estimate that part of the flux that really alters the angular momentum of the stratosphere. In that case,

$$
\phi_{j+(1 / 2)}^{+}(\tau)=\frac{1}{2} \sum_{i}\left[C\left(G_{F},\left(\chi_{i j+1}-\chi_{i j}\right)+\left|\chi_{i j+1}-\chi_{i j}\right|\right) \mid \tau\right]
$$

is the corresponding quantity. Table 3 shows that all belts contribute to $\phi^{+}$at lag $\tau=0$ with maximum values of $6 \mathrm{Ha}^{2}$ near $z \sim 14 \mathrm{~km}$. That means that the total stratosphere would experience an increase of $u_{\mathrm{o}}$ by 1 $\mathrm{m} \mathrm{s}^{-1}$ if this forcing would continue for 10 days. However, as can be seen from Table 3 negative contributions begin to dominate after 3 days. This finding

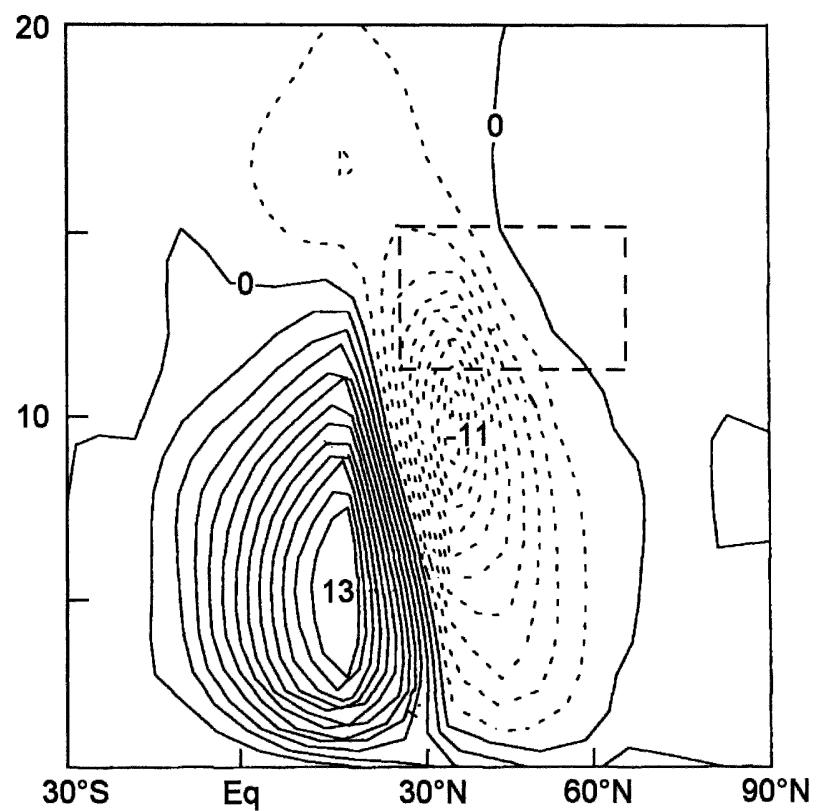

FIG. 4. Streamfunction $\tilde{\psi}$ in $\mathrm{Ha}^{2}$ at lag $\tau=0$ in $\mathrm{M}_{1}$; contour interval $1 \mathrm{Ha}^{3}$; negative values dashed. Rectangle: forcing domain $\mathrm{F}$. 

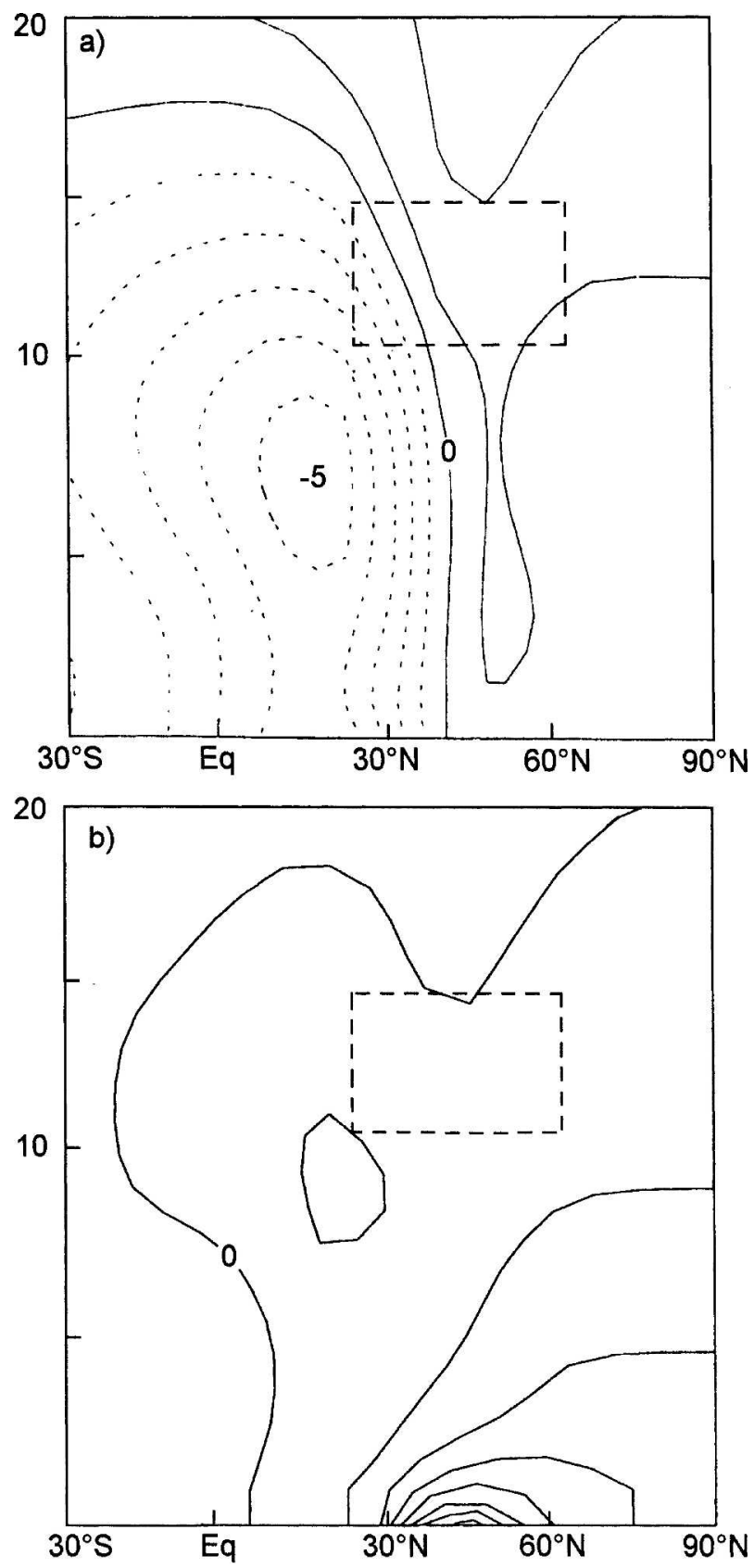

FIG. 5. Velocity potential $\tilde{\chi}$ in $\mathrm{Ha}^{2}$ for $G_{F}$ as prescribed in $\mathrm{M}_{1}$; (a) lag $\tau=0$; (b) lag $\tau=3$ days. Negative values dashed; contour interval $1 \mathrm{Ha}^{2}$. Rectangle: forcing domain $\mathrm{F}$.

reflects the rapid change of the $\chi$ field with lag (see Figs. 5a,b). As can be seen from Fig. 5, however, the domains of strong flux convergence constitute only a small part of the stratosphere. Effects on the local distribution of angular momentum are correspondingly larger.

A shift in the forcing area $\mathrm{F}$ to larger heights does not result in dramatic changes. The overall structure of the
TABLE 2. Total positive and negative vertical flux covariance $F_{j}^{+}(\tau)$ and $F_{j}^{-}(\tau)$ in $\mathrm{Ha}^{2}$ for various lags in at the levels indicated in the first column in $\mathrm{M}_{1}$; heights $z$ in $\mathrm{km}$.

\begin{tabular}{lcrccc}
\hline$\tau$ (days) & -4 & -2 & 0 & 2 & 4 \\
\hline$z=9$ & $75 /-76$ & $92 /-94$ & $240 /-245$ & $76 /-76$ & $33 /-32$ \\
$z=10$ & $58 /-59$ & $79 /-63$ & $203 /-210$ & $68 /-72$ & $27 /-27$ \\
$z=11$ & $37 /-39$ & $41 /-47$ & $150 /-162$ & $57 /-63$ & $26 /-27$ \\
$z=12$ & $24 /-27$ & $25 /-31$ & $97 /-110$ & $42 /-48$ & $22 /-24$ \\
$z=13$ & $17 /-20$ & $15 /-19$ & $54 /-64$ & $26 /-30$ & $17 /-18$ \\
$z=14$ & $12 /-14$ & $9 /-11$ & $27 /-32$ & $17 /-19$ & $13 /-13$ \\
\hline
\end{tabular}

covariances changes little in $\mathrm{M}_{2}$ where $14 \leq z \leq 17 \mathrm{~km}$ but the amplitudes decrease. For example, the minimum of the covariance of the global torque and $G_{F}$ is just $-4 \mathrm{Ha}^{2}$ in this case but there are still positive values for $\tau \geq 10$ days (Fig. 3). Note that there is no shift of the occurrence of the minimum in time. One might expect to see that the minimum is retarded in $\mathrm{M}_{2}$ with respect to $\mathrm{M}_{1}$ because the response to the forcing has to propagate longer to the ground. The absence of this phenomenon indicates again that positive values of $G_{F}$ tend to be embedded in large flow structures and cannot be considered in isolation. The links between stratosphere and troposphere become rather weak when the heights $J_{u}=17, J_{o}=20$ are chosen for the forcing area $\left(\mathrm{M}_{3}\right)$. There is almost no torque in $\mathrm{M}_{3}$ (Fig. 3).

An application of a low-pass-filter-retaining oscillations with periods $\geq 10$ days does not result in fundamental changes. For example, the $\psi$ field at $\tau=0$ has a dipole structure similar to that in Fig. 4. The $\chi$ pattern at $\tau=0$ is smoother than in Fig. 5a but is otherwise similar.

The analysis for that domain in the Southern Hemisphere $\left(63^{\circ} \mathrm{S} \leq \varphi \leq 27^{\circ} \mathrm{S} ; 11 \mathrm{~km} \leq z \leq 14 \mathrm{~km} ; \mathrm{M}_{4}\right)$ that corresponds to $\mathrm{M}_{1}$ gives fairly similar results. For example, the covariance pattern in Fig. 2, when mirrored at the equator, comes quite close to that obtained in $\mathrm{M}_{4}$ except that the minimum there is $-6 \mathrm{Ha}^{2}$. The $\psi$ field at $\tau=0$ has the same double structure as seen in Fig. 4

TABLE 3. Total positive and negative vertical flux covariance $\phi^{+}(\tau), \phi^{-}(\tau)$ at the height levels as indicated in the first column in $\mathrm{M}_{1}$ for three lags.

\begin{tabular}{lccc}
\hline \hline$\tau$ (days) & -3 & 0 & 3 \\
\hline$z=8.5$ & $0.8-0.0$ & $1.2-0.0$ & $1.8-0.4$ \\
$z=9.5$ & $1.1-0.0$ & $2.8-0.0$ & $1.3-0.5$ \\
$z=10.5$ & $1.3-0.0$ & $4.4-0.0$ & $0.9-0.8$ \\
$z=11.11$ & $1.2-0.0$ & $5.6-0.0$ & $0.6-1.0$ \\
$z=12.5$ & $0.9-0.0$ & $6.3-0.0$ & $0.5-1.3$ \\
$z=13.5$ & $0.7-0.0$ & $6.3-0$ & $0.4-1.3$ \\
$z=14.5$ & $0.5-0.0$ & $5.8-0$ & $0.3-1.1$ \\
\hline
\end{tabular}


with an anticyclonic cell in the south but amplitudes are larger with a maximum (minimum) of $191 \mathrm{Ha}^{2}(-272$ $\left.\mathrm{Ha}^{2}\right)$. The related flux covariances $F^{+}(\tau), \phi^{+}(\tau)$ tend also to be larger. For example, the maximum flux $\mathrm{F}^{+}(0)$ is now $380 \mathrm{Ha}^{2}$ and $\phi^{+}(0)$ is as large as $14 \mathrm{Ha}^{2}$.

\section{b. Polar stratosphere}

The reaction of $\tilde{\mu}$ to a shift of $F$ to the latitudes $63^{\circ}-90^{\circ} \mathrm{N}\left(I_{s}=14, I_{n}=17 ; \mathrm{P}_{1}\right)$ reminds us strongly of Fig. 2 (not shown). Again there is this quasibarotropic structure with increase of $\tilde{\mu}$ in the belts north of $\sim 45^{\circ} \mathrm{N}$ with decrease in the south. There is an anticyclonic tropospheric cell extending from the equator to $63^{\circ} \mathrm{N}$ with a weaker cyclonic cell underneath the forcing area much the same as in Fig. 4. The only qualitative dissimilarity is found for the $\chi$ field at lag $\tau=3$ days where the surface friction is a source of angular momentum in the polar region (Fig. 6). An upward displacement of $F$ as in $\mathrm{M}_{2}, \mathrm{M}_{3}$ leads to the same result as at midlatitudes. The reaction is the weaker the higher up the forcing area. All this suggests that we are essentially looking at the same type of events. The analysis for the polar cap in the Southern Hemisphere yields fairly similar results except that amplitudes tend to be larger.

\section{c. Tropical troposphere and stratosphere}

H91 did not look at the situation in the Tropics. Nevertheless, it is of interest to consider this region as well. The forcing area $F$ in the tropical case $T_{1}$ is located in the upper tropical troposphere (see Table 1). The covariance $C\left(G_{F},-D_{m}-D_{w}\right)$ of forcing and flux convergence is displayed in Fig. 7 for lag $\tau=0$. It is seen quite clearly that the concept of a local forcing makes relatively good sense in this case. There is a welldefined maximum of the convergence in the forcing area with smaller values around although there are two minima of smaller amplitude to the north and south (small amplitude). The pattern changes little with lag for $|\tau|<10$ days but maximum amplitudes are just $\leq 0.5$ $\mathrm{Ha}^{2}$ for $|\tau|=8$ days. The covariance $C\left(G_{F}, \tilde{\mu} \mid \tau\right)$ is negative almost throughout the tropical troposphere for $\tau \leq 4$ days but there is the growth of an equatorial column of positive deviations of the angular momentum with negative domains to the north and south. This column is restricted to the lowest $3-4 \mathrm{~km}$ of the troposphere for $\tau=-8$ days to grow to a height of $\sim 15 \mathrm{~km}$ at $\tau=8$ days (Fig. 8). Of course, this increase of $\tilde{\mu}$ is caused by tropospheric flux convergences (see also Fig. $9)$. The related streamfunction $\psi$ is symmetric with respect to the equator with downward fluxes at the equator and upward fluxes to the north and south. The $\chi$ field at $\tau=0$ consists of a well-marked positive center

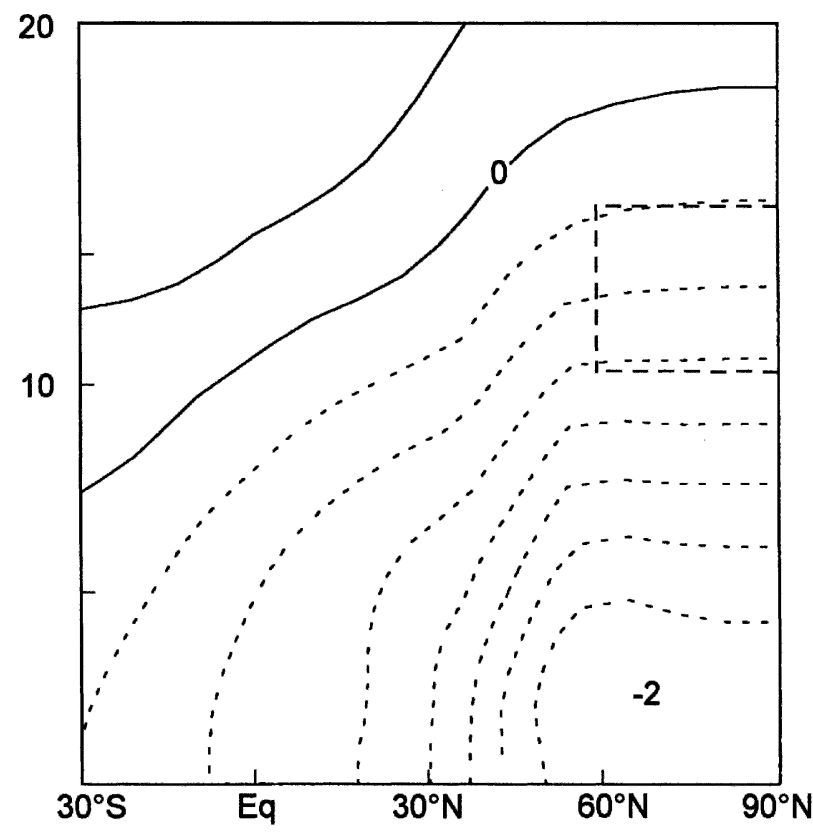

Fig. 6. Velocity potential $\tilde{\chi}$ in $\mathrm{Ha}^{2}$ for $G_{F}$ as prescribed in $\mathrm{P}_{1}$ at lag $\tau=3$ days. Negative values dashes; contour interval $0.25 \mathrm{Ha}^{2}$. Rectangle: forcing domain $\mathrm{F}$.

in the forcing area and negative values throughout the lower troposphere (Fig. 9). There are two frictional source regions. Note the relative maximum of $\chi$ at the equator at all heights. The same structure is seen 3 days

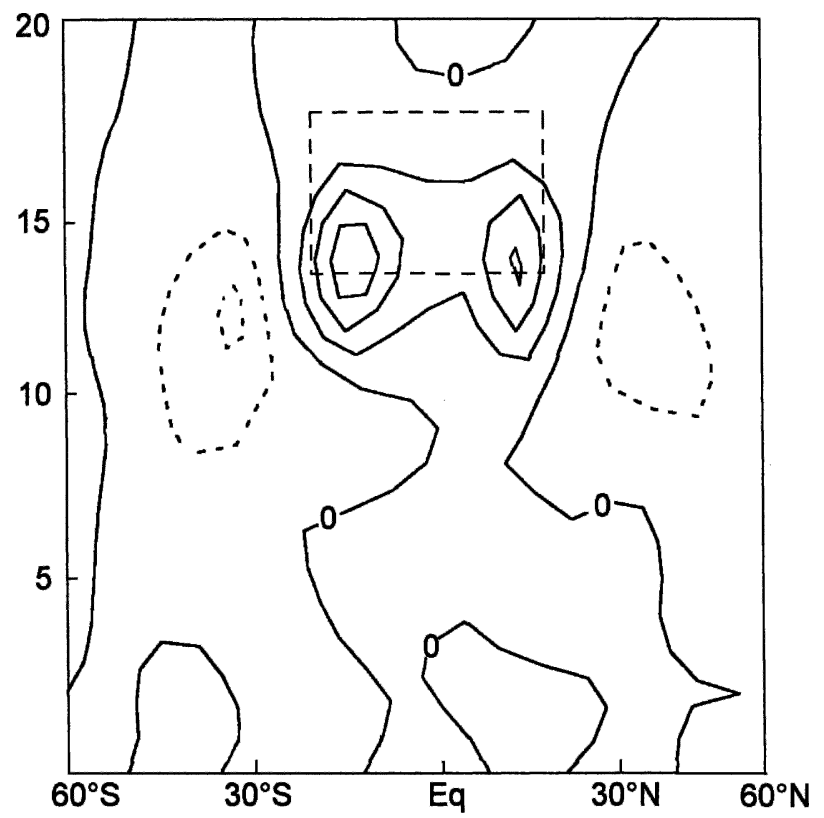

FIG. 7. Covariance function $C\left(G_{F},-D_{w}-D_{m} \mid 0\right)$ of forcing $G_{F}$ in $\mathrm{Ha}^{2}$ in the forcing area $18^{\circ} \mathrm{S} \leq \varphi \leq 18^{\circ} \mathrm{N}, 13 \mathrm{~km} \leq z \leq 16 \mathrm{~km}$ in $\mathrm{Ha}^{2}\left(\mathrm{~T}_{1}\right)$. Negative values dashed; contour interval $1 \mathrm{Ha}^{2}$. Rectangle: forcing domain $\mathrm{F}$. 


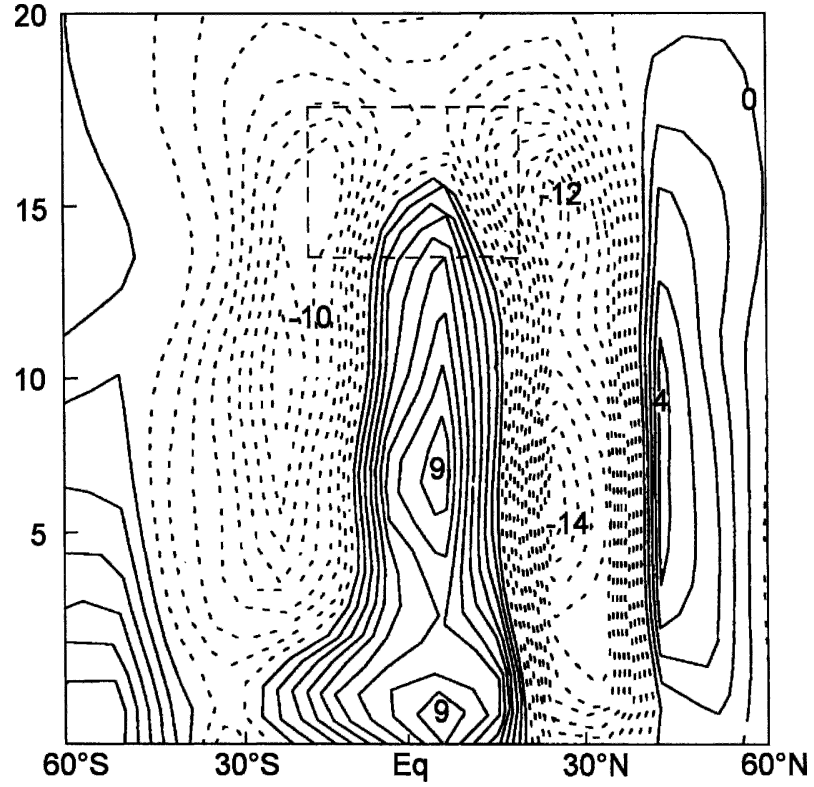

FIG. 8. Covariance function $C\left(G_{F}, \tilde{\mu} \mid 8\right)$ in $\mathrm{T}_{1}$ in $\mathrm{Ha}^{2} \mathrm{~s}^{-1}$. Negative values dashed; forcing area marked by dashed rectangle; contour interval $10 \mathrm{Ha}^{2} \mathrm{~s}^{-1}$.

later. The column of positive angular momentum in Fig. 8 is clearly caused by the convergence of transports from both sides of the equator where the friction torques act as sources. It is the negative anomalies of $\mu$ in the subtropics (see Fig. 8) that induce these torques. The flux $\phi^{+}$is $4 \mathrm{Ha}^{2}$ at $z=8.5 \mathrm{~km}$ and lag $\tau=0$ but

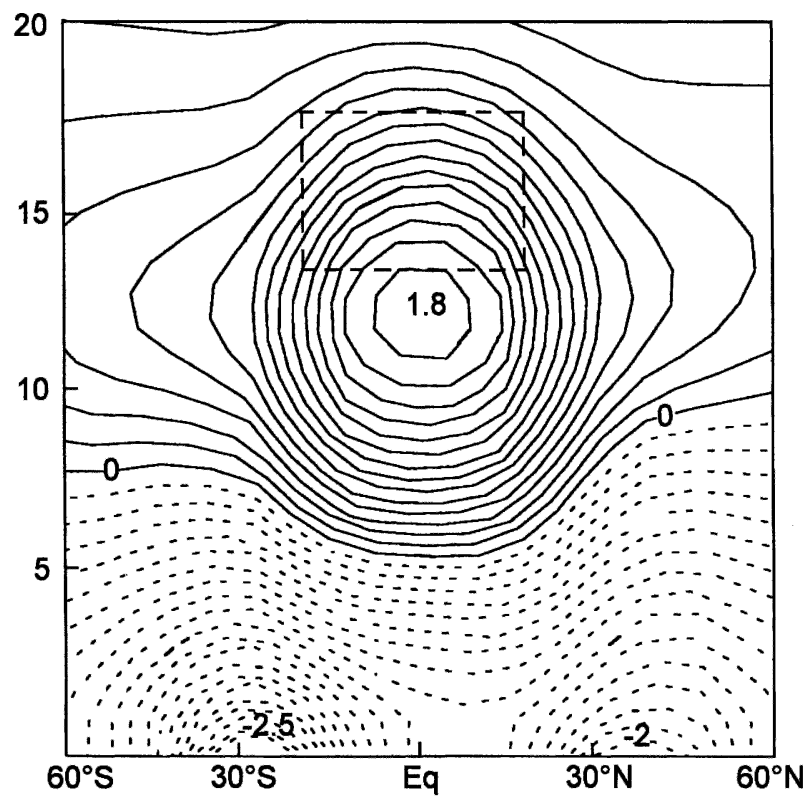

FIG. 9. Covariance function $\tilde{\chi}$ in $\mathrm{Ha}^{2}$ in $\mathrm{T}_{1}$ at lag $\tau=0$ days. Negative values dashed; contour interval $0.1 \mathrm{Ha}^{2}$. Rectangle: forcing domain $\mathrm{F}$. decreases rapidly with height to become negative at $z=$ $14.5 \mathrm{~km}$. In other words, the phenomenon we are looking at is essentially tropospheric. The signals become rather weak and diffuse if we move the forcing area with $16 \mathrm{~km} \leq z \leq 19 \mathrm{~km}$ into the stratosphere. There is still a growth of a tropospheric column of positive angular momentum deviations but the amplitudes are less than one tenth of those in Fig. 7. There are no distinct cells in the $\psi$ field nor is there a clear maximum in the $\chi$ field.

\section{Conclusions and discussion}

Guided by the calculations of H91 an attempt has been made to explore the mean flow response due to upper-level forcing by angular momentum convergence. The lower stratosphere has been chosen as the location of the forcing area $\mathrm{F}$ because data quality there appears to be sufficient for this purpose. H91, as well as other authors, assume an idealized situation where the forcing is due to breaking waves that are excited at a distant location. Moreover, the forcing acts on the mean flow only and is restricted to a certain domain. The response to this forcing has been explored theoretically by H91, Garcia and Boville (1994), Holton et al. (1995), Song and Robinson (2004), and others. It must be expected to occur in the atmosphere as well provided the assumptions of the theory are satisfied. It is clear from the above, however, that this is not the case for the forcing (1.11) due to flux convergence of the wind term in the lower stratosphere. These forcing events are quite short lived. It would be difficult to find the flow evolution presented by H91 on such short times. Moreover, we have been unable to find localized forcing domains outside the Tropics. Instead the eddy forcing in terms of angular momentum convergence tends to extend from the lower stratosphere to the surface (see also Limpasuvan and Hartmann 2000; Lorenz and Hartmann 2001). Areas of positive forcing are always accompanied by neighboring domains of negative forcing as required by the conservation of angular momentum. The $\psi$ field related to such a forcing structure is fairly persistent. It is dominated by downward transports of positive angular momentum at and below the forcing domain with opposite transports to the north and south. The $\chi$ field is more variable in time and describes a momentum flux into the forcing domain at $\tau \sim 0$. For $\tau \sim 3$ days, the role of surface torques becomes obvious. The forcing is better localized in the upper tropical troposphere but can nevertheless not be seen as an isolated event.

Our findings do not exclude the possibility that responses as predicted by $\mathrm{H} 91$ can be observed even in 
the troposphere and lower stratosphere. To detect them, the forcing events would have to be stratified to find events that satisfy the assumptions made in the theories of downward control. As yet, corresponding efforts were not successful. We must stress that we did not look at forcing events above the lower stratosphere. Steady-state aspects of the theory of H91 were excluded a priori. Conservation of angular momentum implies that localized sources due to wave driving must be balanced by sinks. This almost rules out strict localization. Mountain torque events are perhaps an exception because angular momentum is transferred to the atmosphere in that case. Waves may then transport the angular momentum to the forcing region. Egger and Hoinka $(2004,2005)$ demonstrated that the angular momentum transferred during mountain torque events is mainly deposited in the troposphere and the lower stratosphere.

It is a key result that the stratosphere receives angular momentum from the troposphere during these forcing events provided $G_{F}$ is positive. In principle, the angular momentum added in $\mathrm{F}$ could result from the stratosphere itself. However, our results show that a substantial part of this angular momentum is substituted by fluxes from below. In other words, the forcing events constitute events of stratosphere-troposphere exchange with respect to angular momentum.

Acknowledgments. The comments by Y. Song, W. Robinson, and two anonymous referees clearly helped to improve the quality of the text.

\section{REFERENCES}

Andrews, D., J. Holton, and C. Leovy, 1987: Middle Atmosphere Dynamics. Academic Press, 489 pp.

Egger, J., 1996: Comments on "On the 'downward control' of extratropical diabatic circulations by eddy-induced mean zonal forces." J. Atmos. Sci., 53, 2103-2104.
— , and K.-P. Hoinka, 2004: Axial angular momentum: Vertical fluxes and response to torques. Mon. Wea. Rev., 132, 1294 1305.

— and - 2005: Torques and the related meridional and vertical fluxes of axial angular momentum. Mon. Wea. Rev., 133, 621-633.

Feldstein, S., and S. Lee, 1998: Is the atmospheric zonal index driven by an eddy feedback? J. Atmos. Sci., 55, 3077-3086.

Garcia, R., and B. Boville, 1994: "Downward control" of mean meridional circulation and temperature distribution of the polar winter stratosphere. J. Atmos. Sci., 51, 2238-2245.

Gibson, R., P. Kållberg, S. Uppala, A. Hernandes, A. Nomura, and E. Serrano, 1997: ERA description. ECMWF Reanalysis Project Rep. Series, Vol. 1, 86 pp. [Available from ECMWF, Shinfield Park RGZ 9AX United Kingdom.]

Haynes, P., C. Marcks, M. McIntyre, T. Shepherd, and K. Shine, 1991: On the "downward control" of extratropical diabatic circulations by eddy-induced mean zonal forces. J. Atmos. Sci., 48, 651-679.

Hoerling, M., T. Schaack, and A. Lenzen, 1993: A global analysis of stratospheric-tropospheric exchange during northern winter. Mon. Wea. Rev., 121, 162-172.

Holton, J., P. Haynes, M. McIntyre, A. Douglas, R. Rood, and L. Pfister, 1995: Stratosphere-troposphere exchange. Rev. Geophys. Res., 33, 403-439.

Limpasuvan, V., and D. Hartmann, 2000: Wave-maintained annular modes of climate variability. J. Climate, 13, 4414-4429.

Lorenz, D., and D. Hartmann, 2001: Eddy-zonal flow feedback in the Southern Hemisphere. J. Atmos. Sci., 58, 3312-3327.

Papoulis, A., 1965: Probability, Random Variables and Stochastic Processes. MacGraw-Hill, 583 pp.

Shepherd, T., and T. Shaw, 2004: The angular momentum constraint on climate sensitivity and downward control in the middle atmosphere. J. Atmos. Sci., 61, 2899-2908.

Song, Y., and W. Robinson, 2004: Dynamical mechanisms for stratospheric influences on the troposphere. J. Atmos. Sci., 61, 1711-1725.

Wernli, H., and M. Bourqui, 2002: A Lagrangian "1-year climatology" of (deep) cross-tropopause exchange in the extratropical Northern Hemisphere. J. Geophys. Res., 107D, 4021, doi:10.1029/2001JD000812.

Wirth, V., and J. Egger, 1999: Diagnosing extratropical synopticscale stratosphere-troposphere exchange: A case study. Quart. J. Roy. Meteor. Soc., 125, 635-655. 